\title{
Aerodynamic Reduced-Order Modeling without Static Correction Requirement Based on Body Vortices
}

\author{
Suwin Sleesongsom ${ }^{1}$ and Sujin Bureerat ${ }^{2}$ \\ ${ }^{1}$ Department of Mechanical Engineering, Faculty of Engineering, Chiangrai College, Chiangrai 57000, Thailand \\ ${ }^{2}$ Department of Mechanical Engineering, Faculty of Engineering, Khon Kaen University, Khon Kaen 40002, Thailand \\ Correspondence should be addressed to Suwin Sleesongsom; suwins2000@yahoo.com
}

Received 30 December 2012; Revised 25 March 2013; Accepted 25 March 2013

Academic Editor: Sergio Nardini

Copyright (c) 2013 S. Sleesongsom and S. Bureerat. This is an open access article distributed under the Creative Commons Attribution License, which permits unrestricted use, distribution, and reproduction in any medium, provided the original work is properly cited.

\begin{abstract}
The objective of this research is to propose a new reduced-order modeling method. This approach is based on fluid eigenmodes and body vortices without using static correction. The vortex lattice method (VLM) is used to analyze unsteady flows over twodimensional airfoil and three-dimensional wing. Eigenanalysis and reduced-order modeling are performed using a conventional method with static correction and an unconventional one without the static correction. Numerical examples are proposed to demonstrate the performance of the present method. The results show that the new method can be considered an alternative way to perform the reduced-order models of unsteady flow.
\end{abstract}

\section{Introduction}

Reduced-order modeling (ROM) with a static correction technique is an efficient technique which has been used for analyzing unsteady incompressible aerodynamic flows [1]. A few unsteady flow eigenmodes are used to construct the reduced-order unsteady flow models similar to the modal analysis of structures. Such a technique was used to study an eigenanalysis of unsteady potential flows about flat airfoils, cascades, and wings. Later, this technique has been used to analyze a wide variety of unsteady flow models such as a compressor cascade [2] and complex configurations [3]. Unfortunately, conventional ROM without static correction technique cannot generate satisfactory results even with all of eigenmodes [4]. However, the ROM with static correction cannot give a satisfactory result if its time step computed a quasisteady part of the solution. Behbahani-Nejad has shown the eigenmodes that are equal to the body's elements behave exactly in quasistatic fashion [4]. He proposed to construct a ROM only based on the wake eigenmodes, which means that the body quasistatic eigenmodes are removed. Based on such conditions, acceptable results were obtained without using the static correction technique. The new technique is used to solve many fluid flow problems. Shahverdi et al. [5] presented that the new technique can construct an efficient ROM based on the boundary element method to analyze unsteady flows over an airfoil, wing, and wing body. Later, Shahverdi et al. [6] proposed to study an aeroelastic analysis for helicopter rotor blade in hover using the same technique. Dardel and Bakhtiari-Nejad [7] applied the same technique to find a reduced order of complete aeroelastic model for limit cycle oscillations. Their model has significantly low dimension in comparison with other previously studied models.

Another method for constructing ROMs is proper orthogonal decomposition (POD), which has been used for investigating unsteady aerodynamic and aeroelastic models [8]. Kim [9] has used the method for frequency domain of a vortex lattice fluid model. From the literature review of the POD, it has been used to obtain low-dimensional models of fluids [10], but most of the applications have been used for incompressible flows. Later, Rowley [11] proposed a framework for applying the POD and Galerkin projection for compressible fluids. In compressible flow, both the kinematic and thermodynamic variables are dynamically important. They proposed an energy-based inner product used to obtain POD modes. The result was that the POD can be extended to the compressible flow. 
The purpose of this research is to derive an alternative ROM based on a vortex lattice method (VLM) in which the eigenvalue problem is defined only based on the unknown body vortices. The new ROM does not need the static correction as with the previous formulation, which is based on the wake vortices. The eigenvalues of the new eigensystem are nonzero or have no quasistatic eigenmodes. Eigenanalysis results show that the eigenvalues of the proposed method are equal to the corresponding nonzero values of the previous studies. Furthermore, the reduced-order models are constructed for steady flows over a two-dimensional airfoil and a three-dimensional wing. The results show that the present methods can accurately analyze unsteady flows and it is an alternative reduced-order model.

\section{Eigenanalysis and ROM}

The vortex lattice method for unsteady flow (termed as unsteady vortex lattice method (UVLM)) is a relation of downwash and vortex of unsteady flow [1]:

$$
\mathbf{A} \Gamma^{n+1}+\mathbf{B} \Gamma^{n}=\mathbf{w}^{n+1},
$$

where $\boldsymbol{\Gamma}$ is a vector of vortex strength and $\mathbf{w}$ is a vector of downwash. The matrices $\mathbf{A}$ and $\mathbf{B}$ are large sparse matrices. The $\Gamma^{n+1}$ at time $n+1$ can be calculated in terms of the solution at time $n$ by

$$
\Gamma^{n+1}=\mathbf{A}^{-1}\left[\mathbf{w}^{n+1}-\mathbf{B} \Gamma^{n}\right] .
$$

An unsteady lift can be calculated as follows [12]:

$$
L=\int_{-b}^{b} \rho\left[U \gamma(x)+\frac{d}{d t} \int_{-b}^{x} \gamma\left(x_{1}\right) d x_{1}\right] d x,
$$

where $\gamma(x)=d \Gamma / d x$ and $b$ is semichord of the airfoil. For zero downwash, where $\Gamma=x_{i} \exp \lambda_{i} t$ and $z_{i}=\exp \lambda_{i} \Delta t$, the generalized eigenvalue problem can be obtained as

$$
z_{i} A x_{i}+B x_{i}=0
$$

where $z_{i}$ is the $i$ th eigenvalue and $x_{i}$ is the corresponding right eigenvector. More generally, (4) can be written in the form of right eigenvectors $X$ as follows:

$$
\mathbf{A X Z}+\mathbf{B X}=\mathbf{0}
$$

where $\mathbf{Z}$ is a diagonal matrix containing the eigenvalue and $\mathbf{X}$ is a matrix in which columns are the right eigenvectors. Similarly, the eigenvalue problem in the form of left eigenvectors $\mathrm{Y}$ can be written as

$$
\mathbf{A}^{T} \mathbf{Y Z}+\mathbf{B}^{T} \mathbf{Y}=\mathbf{0}
$$

where $\mathrm{Y}$ is a matrix, with the rows being the left eigenvectors. Similar fashion to structural modal analysis, the eigenvectors satisfy the orthogonality conditions

$$
\begin{gathered}
\mathbf{Y}^{T} \mathbf{A X}=\mathbf{I}, \\
\mathbf{Y}^{T} \mathbf{B X}=-\mathbf{Z} .
\end{gathered}
$$

The dynamic behavior of the fluid as the sum of the individual eigenmodes can be expressed as

$$
\Gamma=\mathrm{Xc},
$$

where $\mathbf{c}$ is the vector of normal mode coordinates. Substitution of the (8) into (1), premultiplying by $\mathbf{Y}^{T}$, and using the orthogonality condition give a set of $N$ uncoupled equations for the modal coordinates $\mathbf{c}$,

$$
\mathbf{c}^{n+1}-\mathbf{Z} \mathbf{c}^{n}=\mathbf{Y}^{T} \mathbf{w}^{n+1}
$$

From (9), each mode can be marched forward in time independently. The solutions can then be reassembled using (8) to obtain the vortex strength.

The reduced-order model can be carried out by retaining only a few $m$ of the original modes that give the largest eigenvalues $(m \ll N)$. However, the preceding reduced-order model does not produce satisfactory results due to neglected modes that are not orthogonal to the downwash. The way to solve this problem is to decompose the unsteady solution into two parts. The first part is equivalent to the response of the system if the disturbance is quasisteady and the second part is dynamic part. The unsteady solution is shown as follows:

$$
\Gamma^{n}=\Gamma_{s}^{n}+\bar{\Gamma}^{n}=\Gamma_{s}^{n}+\mathbf{X} \overline{\mathbf{c}}^{n}
$$

The quasisteady portion $\Gamma_{s}$ is given by

$$
[\mathbf{A}+\mathbf{B}] \Gamma_{s}^{n}=\mathbf{w}^{n}
$$

Thus, (9) is replaced by

$$
\overline{\mathbf{c}}^{n+1}-\mathbf{Z} \overline{\mathbf{c}}^{n}=\mathbf{Y}^{T} \mathbf{w}^{n+1}-\mathbf{Y}^{T}\left(\mathbf{A} \Gamma_{s}^{n}+\mathbf{B} \Gamma_{s}^{n}\right) .
$$

\section{ROM without Static Correction Requirement Based on Wake Vortices}

Previous work [5] proposed the method to remove zero eigenvalues by rearranging (1) in such a way that its eigenvalues are the same as the nonzero eigenvalues of the previous equation. The way to solve the problem is to decompose the vortex strength into two parts as body $\left(\boldsymbol{\Gamma}_{b}\right)$ and wake $\left(\boldsymbol{\Gamma}_{w}\right)$ vortex strengths as follows:

$$
\Gamma=\left\{\begin{array}{l}
\Gamma_{b} \\
\Gamma_{w}
\end{array}\right\}
$$

Substitution (13) into (1) and partitioning it lead to

$$
\left[\begin{array}{ll}
\mathbf{A}_{11} & \mathbf{A}_{12} \\
\mathbf{A}_{21} & \mathbf{A}_{22}
\end{array}\right]\left\{\begin{array}{l}
\boldsymbol{\Gamma}_{b} \\
\boldsymbol{\Gamma}_{w}
\end{array}\right\}^{n+1}+\left[\begin{array}{ll}
\mathbf{B}_{11} & \mathbf{B}_{12} \\
\mathbf{B}_{21} & \mathbf{B}_{22}
\end{array}\right]\left\{\begin{array}{l}
\boldsymbol{\Gamma}_{b} \\
\boldsymbol{\Gamma}_{w}
\end{array}\right\}^{n}=\left\{\begin{array}{c}
\mathbf{w}_{b} \\
0
\end{array}\right\}^{n+1}
$$

From the previous equation, it can be arranged as

$$
\begin{gathered}
\mathbf{A}_{11} \boldsymbol{\Gamma}_{b}^{n+1}+\mathbf{A}_{12} \boldsymbol{\Gamma}_{w}^{n+1}+\mathbf{B}_{11} \boldsymbol{\Gamma}_{b}^{n}+\mathbf{B}_{12} \boldsymbol{\Gamma}_{w}^{n}=\mathbf{w}_{b}^{n+1} \\
\mathbf{A}_{21} \boldsymbol{\Gamma}_{b}^{n+1}+\mathbf{A}_{22} \boldsymbol{\Gamma}_{w}^{n+1}+\mathbf{B}_{21} \boldsymbol{\Gamma}_{b}^{n}+\mathbf{B}_{22} \boldsymbol{\Gamma}_{w}^{n}=\mathbf{0} .
\end{gathered}
$$




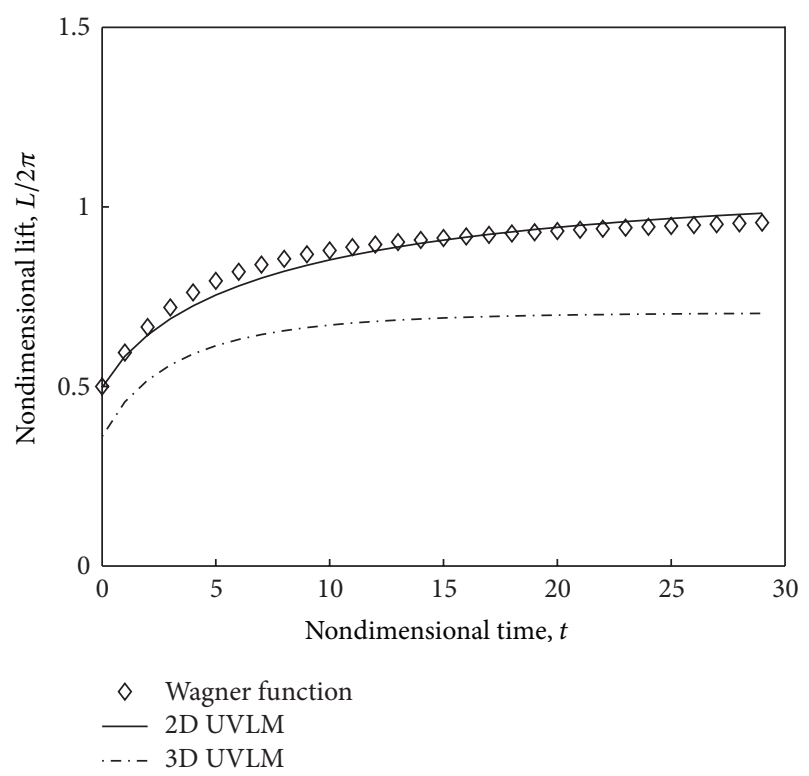

FIgURE 1: Lift acting on the airfoil due to step change in airfoil downwash.

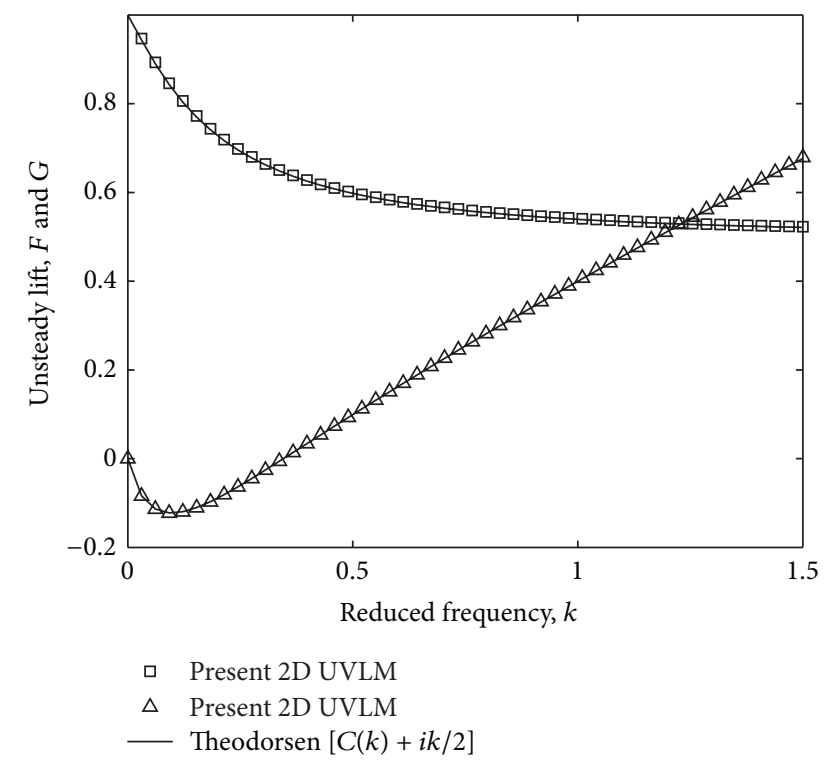

FIGURE 2: Unsteady lift due to plunging motion of an isolated airfoil.

It can be shown that the matrices $\mathbf{B}_{11}$ and $\mathbf{B}_{12}$ are zero; therefore, (15) can be rearranged to a new equation as

$$
\begin{gathered}
\boldsymbol{\Gamma}_{b}^{n+1}=\mathbf{A}_{11}^{-1} \mathbf{w}_{b}^{n+1}-\mathbf{A}_{11}^{-1} \mathbf{A}_{12} \boldsymbol{\Gamma}_{w}^{n+1}, \\
\boldsymbol{\Gamma}_{b}^{n}=\mathbf{A}_{11}^{-1} \mathbf{w}_{b}^{n}-\mathbf{A}_{11}^{-1} \mathbf{A}_{12} \boldsymbol{\Gamma}_{w}^{n} .
\end{gathered}
$$

Substitution of the previous equation into (16) gives

$$
\begin{aligned}
{\left[\mathbf{A}_{22}\right.} & \left.-\mathbf{A}_{21} \mathbf{A}_{11}^{-1} \mathbf{A}_{12}\right] \Gamma_{w}^{n+1}+\left[\mathbf{B}_{22}-\mathbf{B}_{21} \mathbf{A}_{11}^{-1} \mathbf{B}_{12}\right] \Gamma_{w}^{n} \\
& =-\mathbf{A}_{21} \mathbf{A}_{11}^{-1} \mathbf{w}_{b}^{n+1}-\mathbf{B}_{21} \mathbf{A}_{11}^{-1} \mathbf{w}_{b}^{n},
\end{aligned}
$$

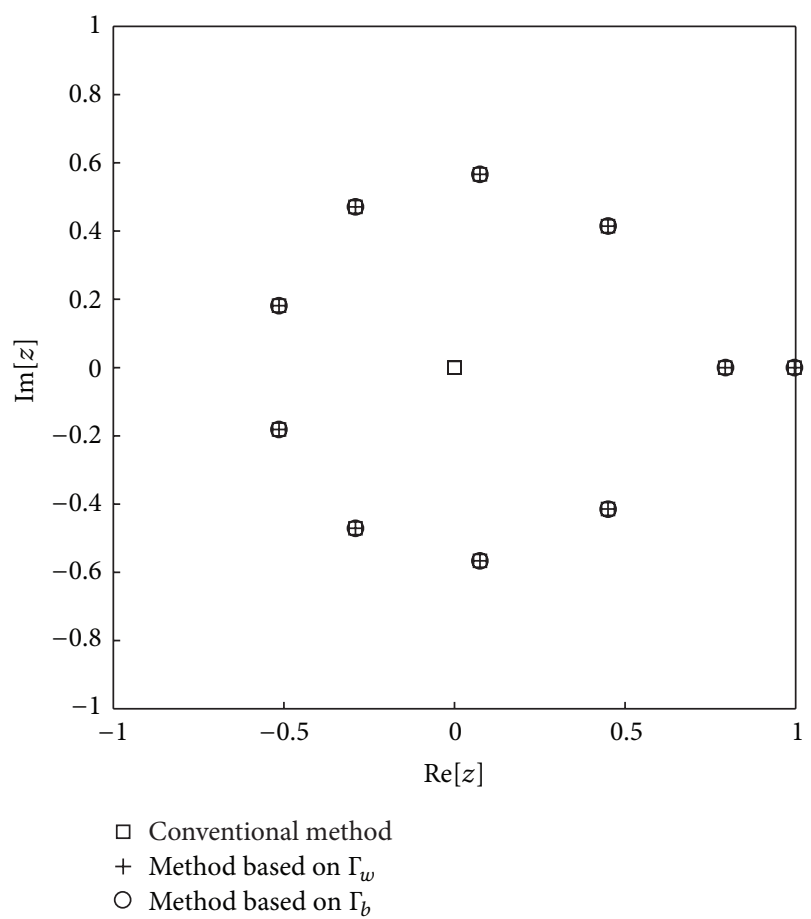

FIGURE 3: Eigenvalues for two-dimensional airfoil.

or

$$
\mathbf{A}_{\text {new }} \boldsymbol{\Gamma}_{w}^{n+1}+\mathbf{B}_{\text {new }} \boldsymbol{\Gamma}_{w}^{n}=\mathbf{w}_{\text {new }}^{n+1}
$$

where

$$
\begin{gathered}
\mathbf{A}_{\text {new }}=\mathbf{A}_{22}-\mathbf{A}_{21} \mathbf{A}_{11}^{-1} \mathbf{A}_{12}, \\
\mathbf{B}_{\text {new }}=\mathbf{B}_{22}-\mathbf{B}_{21} \mathbf{A}_{11}^{-1} \mathbf{B}_{12}, \\
\mathbf{w}_{\text {new }}^{n+1}=-\mathbf{A}_{21} \mathbf{A}_{11}^{-1} \mathbf{w}_{b}^{n+1}-\mathbf{B}_{21} \mathbf{A}_{11}^{-1} \mathbf{w}_{b}^{n} .
\end{gathered}
$$

From (19), which is based on only $\boldsymbol{\Gamma}_{w}$, the corresponding eigensystem has no zero eigenvalues. Therefore, one may construct accurate reduced-order models without using a static correction technique.

\section{Alternative ROM without Static Correction Requirement Based on Body Vortices}

In this section, the authors proposed another way to remove zero eigenvalues by defining a new eigenvalue problem. From (15), with the same reason as the previous section, it can be shown that the matrices $\mathbf{B}_{11}$ and $\mathbf{B}_{12}$ are zero; therefore, (15) can be rearranged to a new equation as follows:

$$
\begin{gathered}
\boldsymbol{\Gamma}_{w}^{n+1}=\mathbf{A}_{12}^{-1} \mathbf{w}_{b}^{n+1}-\mathbf{A}_{12}^{-1} \mathbf{A}_{11} \boldsymbol{\Gamma}_{b}^{n+1}, \\
\boldsymbol{\Gamma}_{w}^{n}=\mathbf{A}_{12}^{-1} \mathbf{w}_{b}^{n}-\mathbf{A}_{12}^{-1} \mathbf{A}_{11} \boldsymbol{\Gamma}_{b}^{n} .
\end{gathered}
$$

Substitution of the previous equation into (16) gives

$$
\begin{aligned}
{\left[\mathbf{A}_{21}\right.} & \left.-\mathbf{A}_{22} \mathbf{A}_{12}^{-1} \mathbf{A}_{11}\right] \mathbf{\Gamma}_{b}^{n+1}+\left[\mathbf{B}_{21}-\mathbf{B}_{22} \mathbf{A}_{12}^{-1} \mathbf{A}_{11}\right] \boldsymbol{\Gamma}_{b}^{n} \\
& =-\mathbf{A}_{22} \mathbf{A}_{12}^{-1} \mathbf{w}_{b}^{n+1}-\mathbf{B}_{22} \mathbf{A}_{12}^{-1} \mathbf{w}_{b}^{n},
\end{aligned}
$$




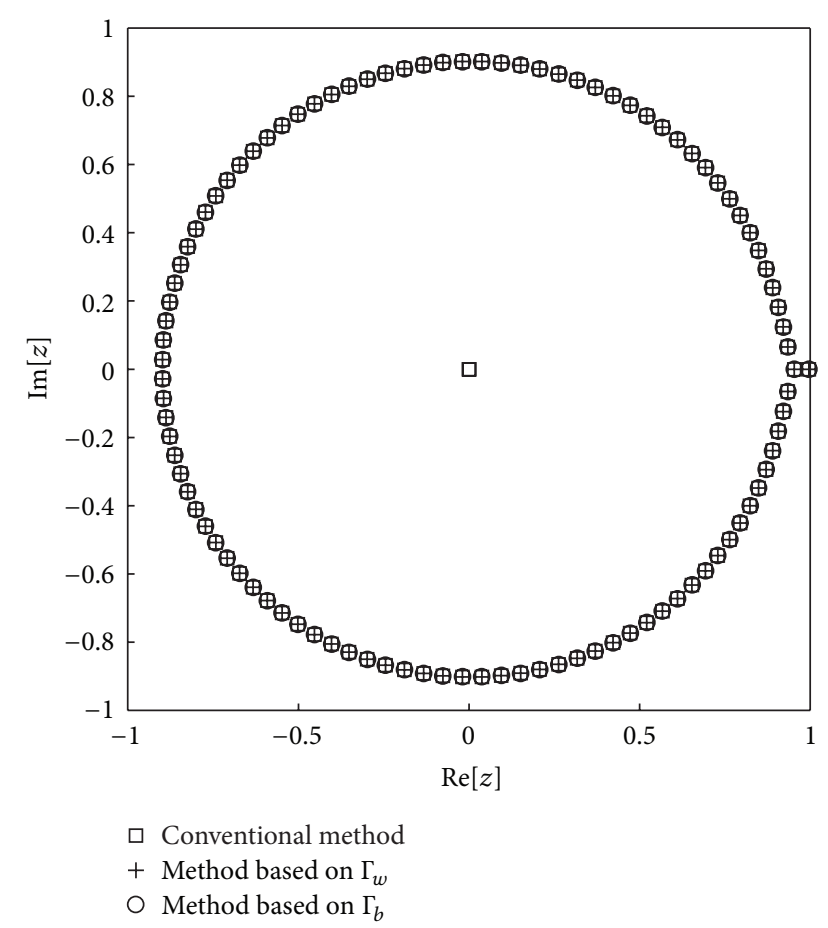

FIGURE 4: Eigenvalues for two-dimensional airfoil when increasing the number of element panels.

or

$$
\mathbf{A}_{\text {new }} \boldsymbol{\Gamma}_{b}^{n+1}+\mathbf{B}_{\text {new }} \boldsymbol{\Gamma}_{b}^{n}=\mathbf{w}_{\text {new }}^{n+1}
$$

where

$$
\begin{gathered}
\mathbf{A}_{\text {new }}=\mathbf{A}_{21}-\mathbf{A}_{22} \mathbf{A}_{12}^{-1} \mathbf{A}_{11}, \\
\mathbf{B}_{\text {new }}=\mathbf{B}_{21}-\mathbf{B}_{22} \mathbf{A}_{12}^{-1} \mathbf{A}_{11}, \\
\mathbf{w}_{\text {new }}^{n+1}=-\mathbf{A}_{22} \mathbf{A}_{12}^{-1} \mathbf{w}_{b}^{n+1}-\mathbf{B}_{22} \mathbf{A}_{12}^{-1} \mathbf{w}_{b}^{n} .
\end{gathered}
$$

From (23), which is based on only $\Gamma_{b}$, the corresponding eigensystem has no zero eigenvalue, which is similar to the previous method. Therefore, we construct the accurate reduced-order models without using static correction technique. Note that since to matrix $\mathbf{A}_{12}$ can be a nonsquare matrix, its inverse cannot be found. This can be solved by using the same numbers of elements for wing panels and wakes.

\section{Results and Discussion}

5.1. Our Vortex Lattice Model. In this section, we validate unsteady vortex lattice model with two unsteady flow models. The first model is the lift acting on the airfoil due to a step change in airfoil downwash (Wagner problem). The result is shown in Figure 1, and our vortex lattice model results are in agreement with the Wagner function. Figure 1 shows the indicial response of a rectangular wing due to rigid body plunging motion in comparison with the corresponding results presented in $[1,4]$. The wing model has aspect ratio of

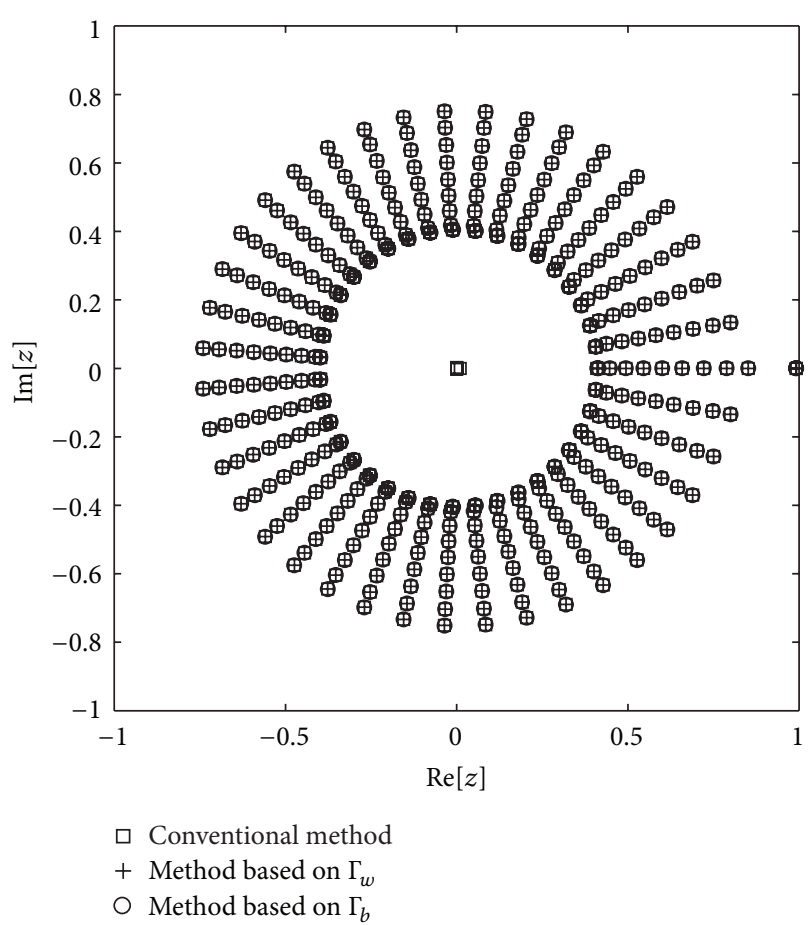

FIGURE 5: Eigenvalues of vortex lattice model of unsteady flow about the three-dimensional wing $-\square$ conventional method, + method based on $\Gamma_{w}$, and o present method.

5.0 and it is modeled with 8 vortex elements in the streamwise direction and 10 element panels in the spanwise direction, while the wake is modeled with 40 vortex elements in streamwise direction. The vortex relaxation factor of this model is 0.992. Also shown in Figure 1, the results of our threedimensional vortex lattice model are in good agreement with $[1,4]$. For the second model, the unsteady lift due to plunging motion of an isolated airfoil is computed for a range of reduced frequencies and compared with the Theodorsen exact solution as shown in Figure 2. The airfoil is modeled using 20 vortex elements, while the wake is model with 200 vortex elements. The vortex relaxation factor is 0.996 . The results in Figure 2 show our model is quite satisfactory with the exact solution.

5.2. Eigenanalysis. This section proposed the eigenanalysis of the conventional method, the method based on $\Gamma_{w}$, and the present method (based on $\Gamma_{b}$ ). The eigenvalues of the twodimensional airfoil are shown in Figure 3. Both airfoil and wake are modeled using 10 vortex elements. The results show that the eigenvalues of the proposed method are the same as the nonzero eigenvalues of the conventional method and the method based on $\Gamma_{w}$. In the proposed eigenanalysis, the eigensystem is interpreted only by the wake elements [4].

Eigenvalues of vortex lattice model of unsteady flow about the two dimensional airfoil and wake are modeled with 100 vortex elements are shown in Figure 4.

Eigenvalues of vortex lattice model of unsteady flow about the three-dimensional wing are plotted in Figure 5. From the figure, the nonzero eigenvalues of the conventional method 


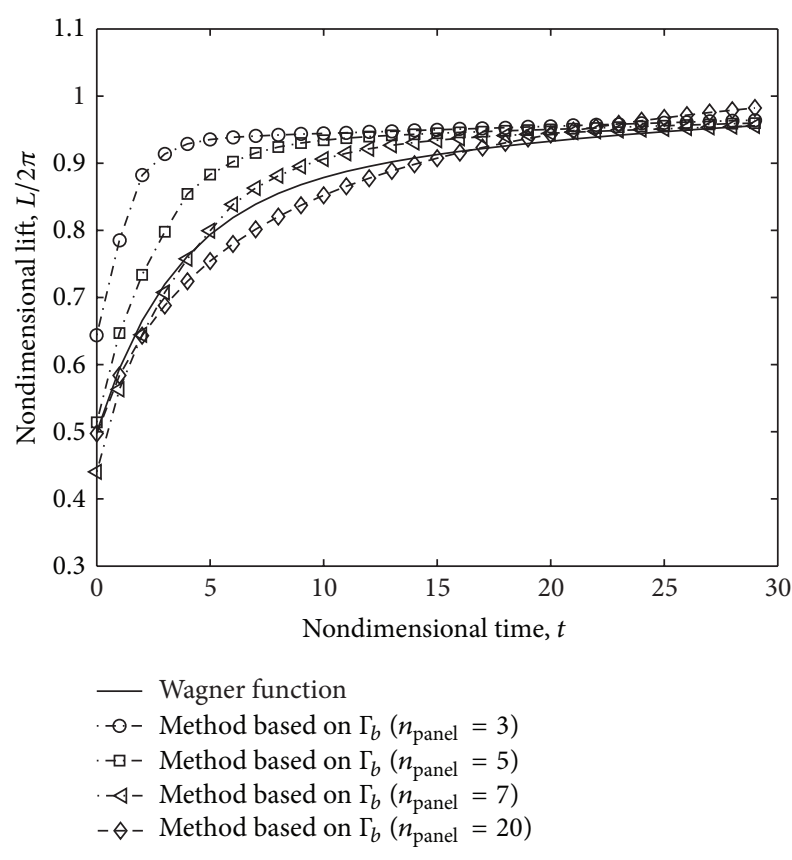

FIGURE 6: Unsteady lift for two-dimensional airfoil predicted using ROM based on $\Gamma_{b}$.

and the method based on $\Gamma_{w}$ are the same with the eigenvalues of the present method.

5.3. Reduced-Order Models. In this section, we used the eigenvalues, which have been studied in the previous section to construct a reduced-order aerodynamic model. The present ROM is called new ROM based on $\Gamma_{b}$. The accuracy of the proposed method is shown in Figures 6 and 7. The first figure (Figure 6) shows the unsteady lift on isolated flat plate airfoil due to step change in airfoil downwash (Wagner problem) predicted using different number of element panels as $3,5,7$, and 20 . The result shows that the present ROM can produce satisfactory results when increasing of element panels. Figure 7 shows the unsteady lift of the airfoil predicted using different number of element panel as 3,5, 7, and 20. The results show that the present ROM can produce satisfactory results when increasing of element panels. Both models can be properly predicted by the present ROM.

\section{Conclusions and Future Work}

In the present work, a reduced-order model for unsteady flows is developed without a static correction technique. This method is proposed to construct the eigensystem based on body vortices, and the rank of the eigensystem is lower than the corresponding eigensystem of the conventional method and the method based on wake vortices. Furthermore, the eigensystem does not contain any zero eigenvalues, so the static correction is not needed. The results show that the proposed method can produce satisfactory results like the conventional method and the method based on wake vortices. For the future work, we plan to apply a pseudoinverse

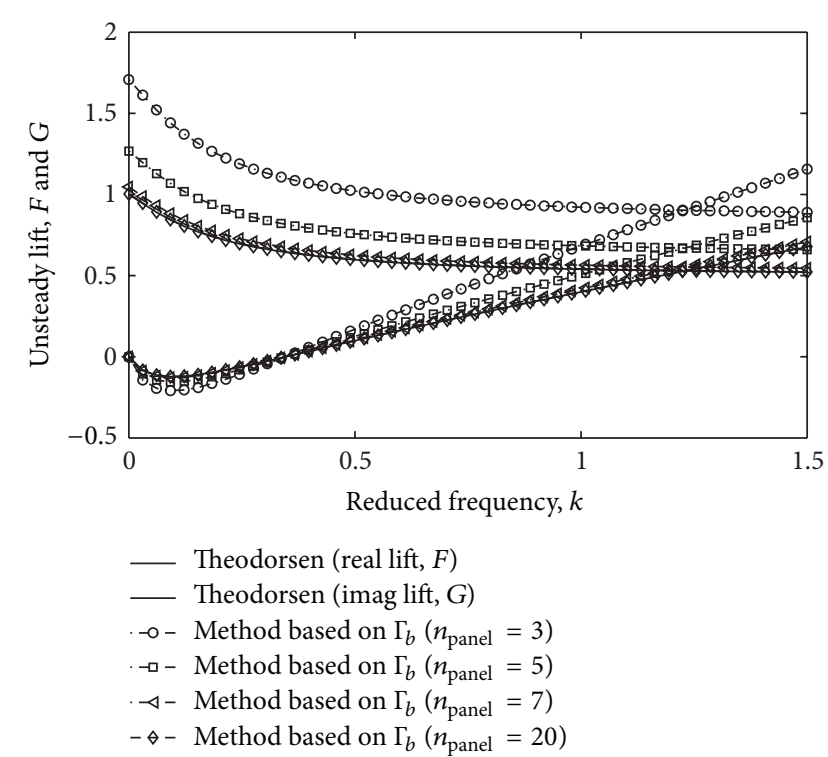

Figure 7: Lift acting on the airfoil due to step change in airfoil downwash predicted using ROM based on $\Gamma_{b}$.

operator to solve nonsquare matrix $\mathrm{A}_{12}$ and then extend the model for aeroelastic analyses of wings.

\section{Acknowledgment}

The authors are grateful for the financial support provided by Chiangrai College.

\section{References}

[1] K. C. Hall, "Eigenanalysis of unsteady flows about airfoils, cascades, and wings," AIAA Journal, vol. 32, no. 12, pp. 24262432, 1994.

[2] R. Florea, K. C. Hall, and P. G. A. Cizmas, "Reduced-order modeling of unsteady viscous flow in a compressor cascade," AIAA Journal, vol. 36, no. 6, pp. 1039-1048, 1998.

[3] V. Esfahanian and M. Behbahani-nejad, "Reduced-order modeling of unsteady flows about complex configurations using the boundary element method," Journal of Fluids Engineering, vol. 124, no. 4, pp. 988-993, 2002.

[4] M. Behbahani-Nejad, H. Haddadpour, and V. Esfahanian, "Reduced-order modeling of unsteady flows without static correction requirement," Journal of Aircraft, vol. 42, no. 4, pp. 882-886, 2005.

[5] H. Shahverdi, A. S. Nobari, M. Behbahani-Nejad, and H. Haddadpour, "An efficient reduced-order modelling approach based on fluid eigenmodes and boundary element method," Journal of Fluids and Structures, vol. 23, no. 1, pp. 143-153, 2007.

[6] H. Shahverdi, A. S. Nobari, M. Behbahani-Nejad, and H. Haddadpour, "Aeroelastic analysis of helicopter rotor blade in hover using an efficient reduced-order aerodynamic model," Journal of Fluids and Structures, vol. 25, no. 8, pp. 1243-1257, 2009.

[7] M. Dardel and F. Bakhtiari-Nejad, "A reduced order of complete aeroelastic model for limit cycle oscillations," Aerospace Science and Technology, vol. 14, no. 2, pp. 95-105, 2010. 
[8] M. C. Romnowski, "Reduced order unsteady aerodynamic and aerolastic models using Karhunen-Loeve eigenmodes," AIAA Paper 96-3981, 1996.

[9] T. Kim, "Frequency-domain Karhunen-Loeve method and its application to linear dynamic systems," AIAA Journal, vol. 36, no. 11, pp. 2117-2123, 1998.

[10] E. H. Dowell, K. C. Hall, J. P. Thomas, R. Florea, B. I. Epureanu, and J. Heeg, "Reduced order models in unsteady aerodynamics," AIAA Paper 99-1261, 1999.

[11] C. W. Rowley, T. Colonius, and R. M. Murray, "Model reduction for compressible flows using POD and Galerkin projection," Physica D, vol. 189, no. 1-2, pp. 115-129, 2004.

[12] J. Katz and A. Plotkin, Low-Speed Aerodynamics from Wing Theory to Panel Methods, MCgraw-Hill, Singapore, 1991. 

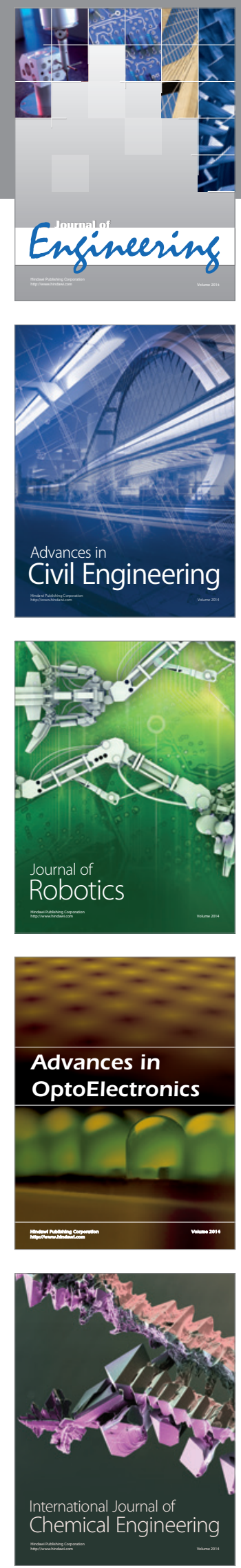

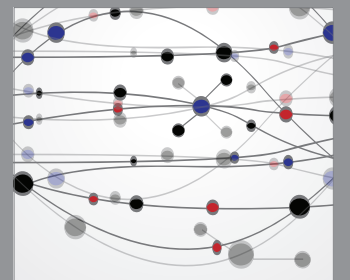

The Scientific World Journal
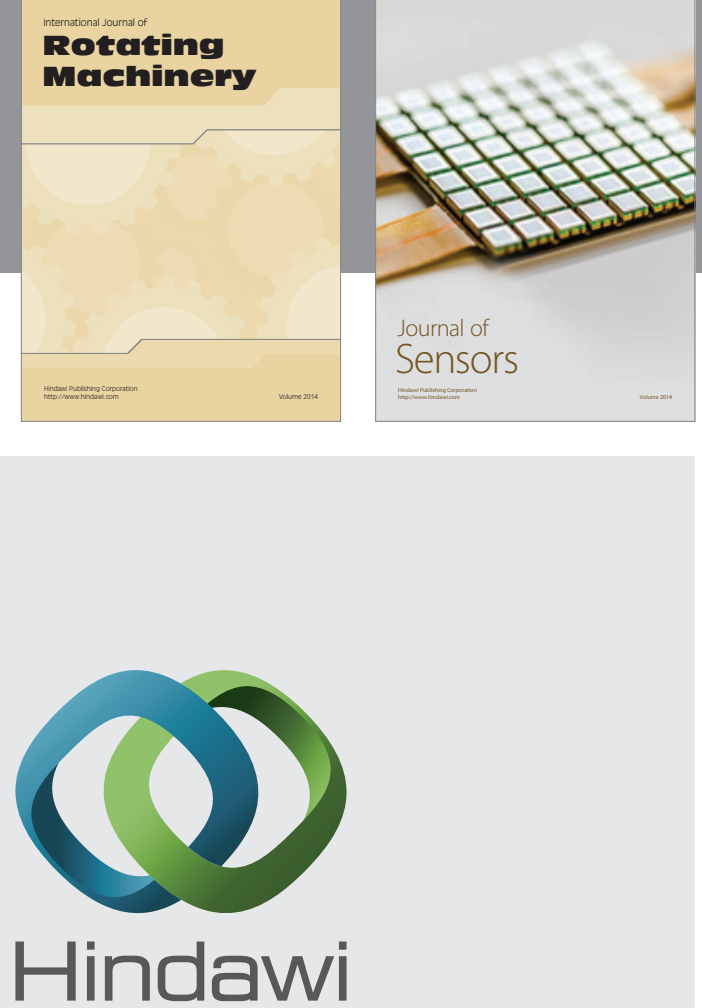

Submit your manuscripts at http://www.hindawi.com
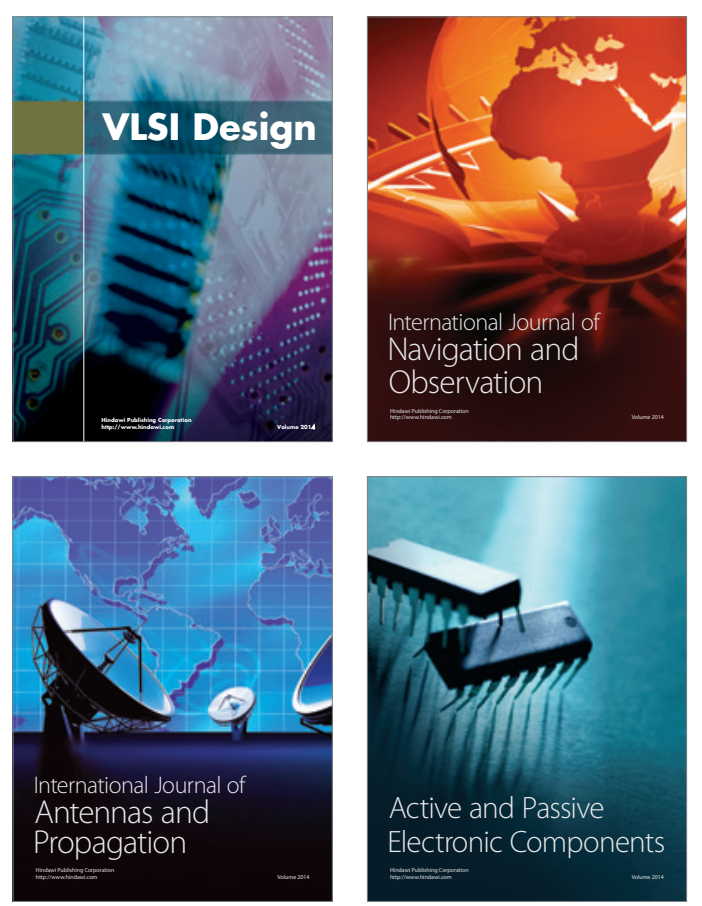
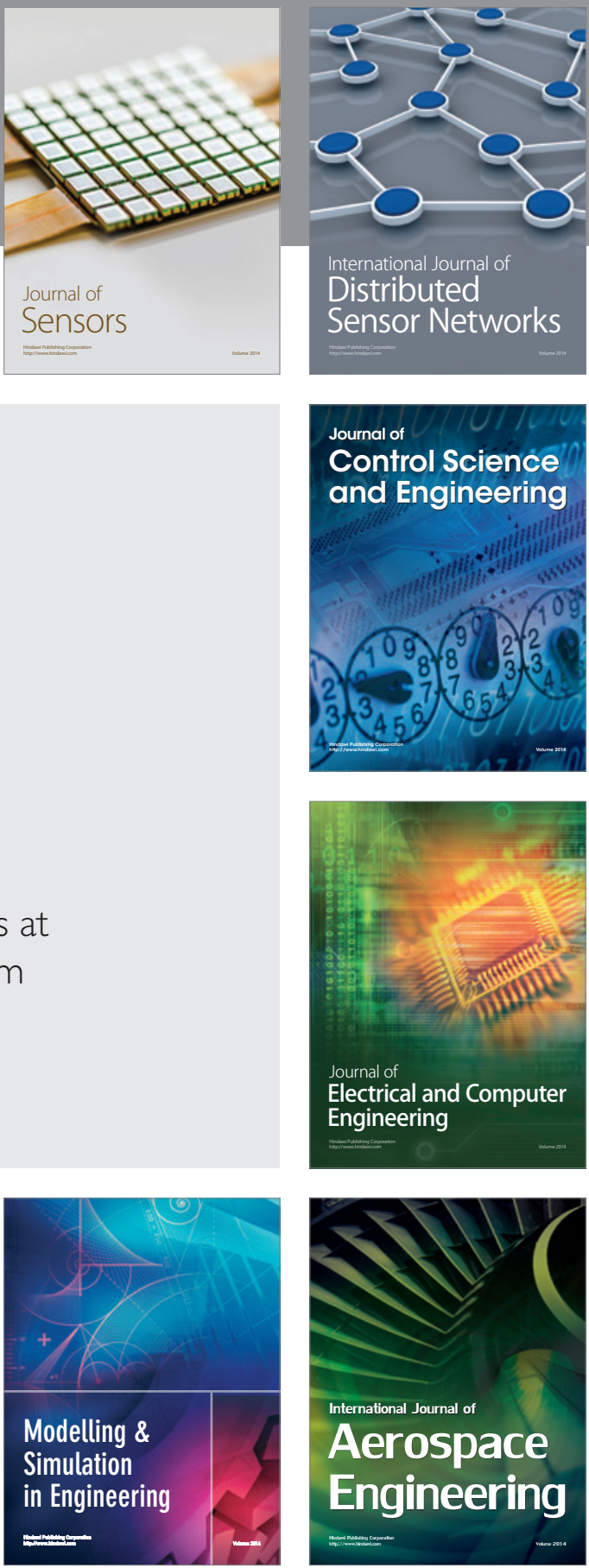

Journal of

Control Science

and Engineering
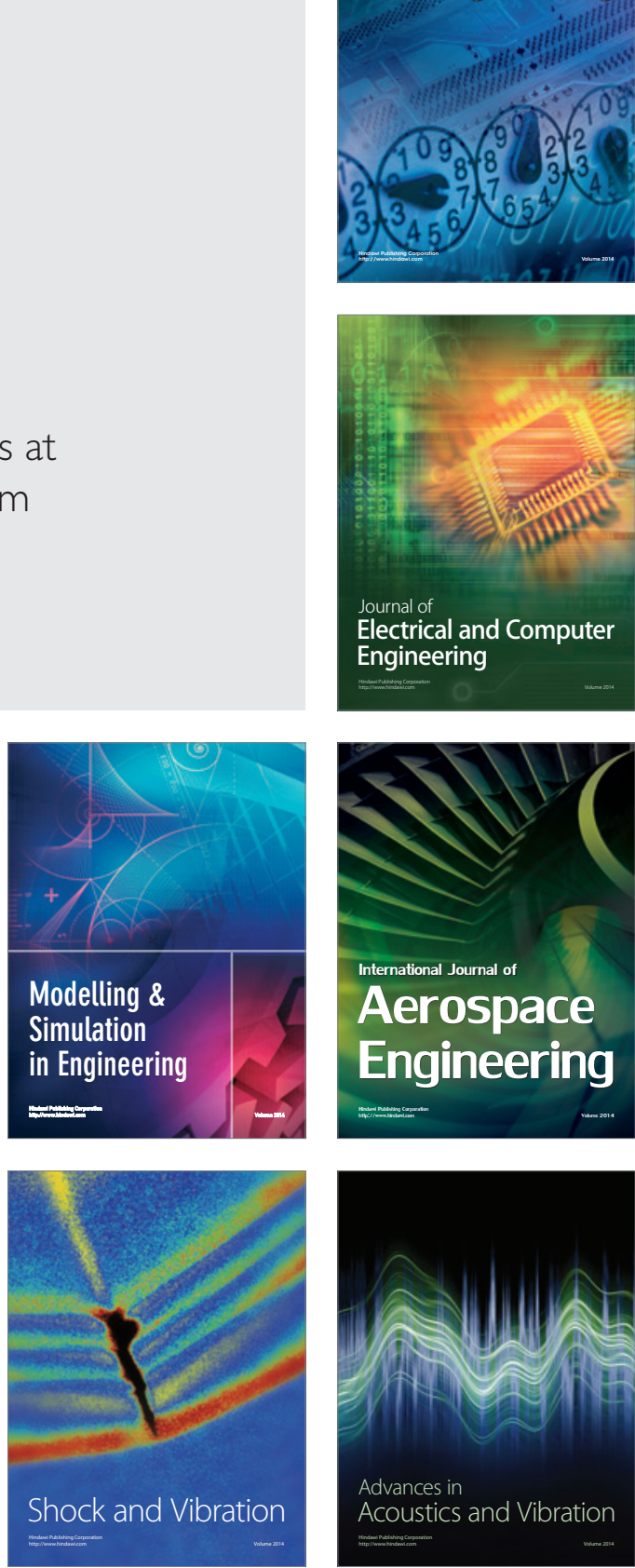